peqGold V

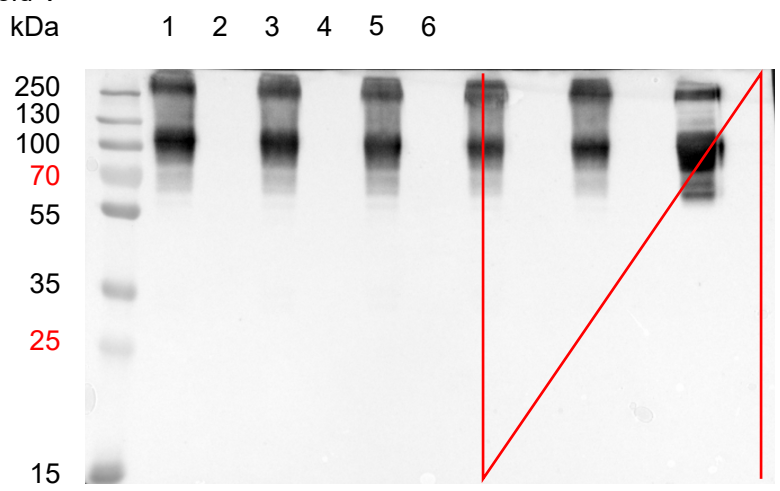

\title{
Image Report:
}

\section{Acquisition Information}

\begin{tabular}{|l|l|}
\hline Imager & ChemiDoc ${ }^{\mathrm{TM} M P}$ \\
\hline Exposure Time $(\mathrm{sec})$ & 4.000 (Manual) \\
\hline Flat Field & Applied (Lens) \\
\hline Serial Number & 731 BR00318 \\
\hline Software Version & 4.0 .1 \\
\hline Application & Chemi Hi Resolution \\
\hline Excitation Source & No Illumination \\
\hline Emission Filter & No Filter \\
\hline Binning & $2 \times 2$ \\
\hline
\end{tabular}

Image Information

\begin{tabular}{|l|l|}
\hline Acquisition Date & $09.08 .201210: 33: 39$ \\
\hline User Name & annawerner \\
\hline Image Area (mm) & X: 150.0 Y: 112.1 \\
\hline Pixel Size (um) & X: 215.5 Y: 215.5 \\
\hline Data Range (Int) & $0-62648$ \\
\hline
\end{tabular}

\section{Analysis Settings}

No analysis performed

S2 Fig. Uncropped image of the Western analysis of SERT. Provided is the original uncropped image from which the bands of the lower panel in the main figure 3 were excised. Included are the parameters describing the image acquisition as documented by the imaging system (ChemiDoc from BioRad). The image contains more lanes than were used for this manuscript, numbered (1-6) are the ones extracted for figure 3 . 